ing to be equivalent). It seems an unnecessary complication to formalize this with new symbols and nomenclature which do little more than obscure the physical basis of the phenomena.

I wish to thank Dr H. G. Midgley for providing the material; Prof. J.D. Bernal for his encouragement and advice; Dr F.M. Lea for permission to publish the results of work which was partly carried out under a programme of extra-mural research for the Building Research Board; and Dr H. F. W. Taylor for assistance in the early stages of the work.

\section{References}

BarnICK, A. W. (1936). Mitt. K.-Wilh.-Inst. Silikatforsch. No. 172. (Abstracted in Strukturbericht, 4, 207.)

Buerger, M. J. (1945). Amer. Min. 30, 469.

Ewald, P. P. (1940). Proc. Phys. Soc. 52, 167.

Iто, T. (1950). X-ray Studies on Polymorphism. Tokyo: Maruzen.

Peacock, M. A. (1935a). Amer. J. Sci. 30, 495.

Peacock, M. A. (1935b). Z. Krystallogr. 90, 97.

Warren, B. E. \& Biscoe, J. (1931). Z. Krystallogr. 80, 391.

WILSON, A.J.C. (1949). X-ray Optics, p. 49. London: Methuen.

Acta Cryst. (1953). 6, 825

\title{
The Multiple-Film Technique: the Effect of Angle of Incidence on the Correlating Factor
}

\author{
By G.J. BULleN \\ Department of Chemical Crystallography, University College, London W.C. 1, England
}

(Received 25 May 1953 and in revised form 17 June 1953)

\begin{abstract}
It is noted that the correlating factor for the multiple-film technique depends on the angle of incidence of the diffracted beam on the film and so has not the same value for all reflexions. The variation of the factor is discussed in detail for oscillation photographs recorded on cylindrical films with the axis of the cylinder as the axis of rotation of the crystal, the factor being constant for all reflexions on the same layer line. Methods of using spots on all layer lines, in an averaging process for finding the factor for each layer, are suggested. The treatment is also applicable to the method of interleaving films with metal foil when using Mo $K$ radiation.
\end{abstract}

The multiple-film technique first described by Robertson (1943) is nowadays widely used for the measurement of intensities by the visual method. The intensities of the spots on the second and succeeding films are related to those of the spots on the first film by a correlating factor for which Robertson gave a value of about 2 , for $\mathrm{Cu} K \alpha$ radiation at normal incidence. The variation, with angle of incidence of the diffracted beam on the film, of absorption and photographic action in a single film, as applying to intensity measurements, has been considered by Cox \& Shaw (1930) and, more recently, by Whittaker (1953). Whereas the variation of photographic action does not affect the multiple-film technique, the variation of absorption does, causing a change in the correlating factor.*

To discuss this change, let us consider an oscillation photograph recorded on cylindrical films with the axis of the cylinder as the axis of rotation of the crystal. If $I_{0}$ is the intensity recorded on the first (i.e. nearest to crystal) film and $I$ that on the second $\left(I_{0}\right.$ and $I$ being within the range of reasonably accurate intensity measurement, so that reflexions giving either very strong or imperceptible blackening of

* Note added in proof, 24 September 1953.- Since the sub. mission of this paper, this effect has been mentioned by Qurashi (1953). either film must be neglected), we have, using double emulsion films,

$$
\left(I / I_{0}\right)_{n}=\exp \left[-\left(2 \mu_{e} t_{e}+\mu_{b} t_{b}\right) \sec \nu_{n}\right],
$$

where the subscript $n$ denotes the $n$th layer line; $\mu_{e}$ and $\mu_{b}$ are the linear absorption coefficients of the emulsion and base respectively; $t_{e}$ and $t_{b}$ are the thicknesses of one side of the emulsion and of the base respectively; and $v_{n}$ is the inclination of the diffracted beam to the equatorial layer.

For the zero layer,

$$
\left(I / I_{0}\right)_{0}=\exp \left[-\left(2 \mu_{e} t_{e}+\mu_{b} t_{b}\right)\right],
$$

and thus, inverting,

$$
\left(I_{0} / I\right)_{n}=\left(I_{0} / I\right)_{0}^{\sec v_{n}}=\left(I_{0} / I\right)_{0}^{\alpha},
$$

where $\alpha=\sec \nu_{n}=\left(1-\zeta^{2}\right)^{-\frac{1}{2}}$.

Let $\left(I_{0} / I\right)_{n}=R_{n}$, the correlating factor for the $n$th layer.

Then

$$
R_{n}=R_{0}^{\alpha} .
$$

The effect is considerable for high layer lines, as $\alpha$ increases to 1.7 when $\zeta=0.8$, and is by no means negligible even for lower layers. If the intensity measurements are to be at all accurate, it must be taken into account. 


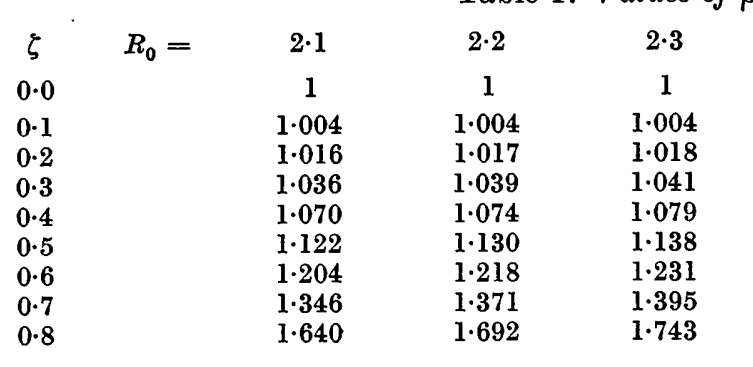

We wish to know $R_{n}$ for each layer line and we can calculate it from $R_{0}$ since we know $\alpha$. To find $R_{0}$, it is best to average the measured ratios for a large number of spots distributed over the whole film. However, the measured ratios give $R_{0}, R_{1}, R_{2}, \ldots$, and, in order to average them, they must be reduced to the corresponding $R_{0}$. There are several ways of accomplishing this:

(a) $R_{n}$ can be found for each layer and the $\alpha$ th root taken. The values of $\stackrel{\alpha}{V} R n\left(=R_{0}\right)$, suitably weighted for the number and intensities of spots used for each, can then be averaged. This involves tedious calculation, but it must be used where method (c) described below fails.

(b) Graphical methods, such as plots of $R_{n}$ against $\zeta$, $\log R_{n}$ against $\alpha$ and $\log \log R_{n}$ against $\log \alpha$, suggest themselves but are found to be inaccurate in practice.

(c) This method is of special applicability to the case of $\mathrm{Cu} K \alpha$ radiation for which $R_{0}$ is about $2 \cdot 3$ for Ilford Industrial $G$, double-emulsion X-ray film. When $R_{0}$ has such a value, which is small compared with that for radiations of longer wavelength (and one would probably not use the multiple-film technique for long-wavelength radiation because of the high correlating factor), we may use

$$
R_{n}=\beta_{n} R_{0},
$$

where $\beta_{n}$ varies with $n$ but is almost independent of $R_{0}$. This is only an empirical relation but it is true for the range of values of $R_{0}$ encountered. $\beta_{n}$ can be calculated theoretically for various values of $R_{0}$ by combining equations (4) and (5), whence

$$
\beta_{n}=R_{0}^{\alpha-1} \text {. }
$$

Some values of $\beta_{n}$ are given in Table 1 .

From this table, it is seen that $\beta_{n}$ may be considered to be independent of $R_{0}$, the greatest variation (when $\zeta=0.8$ ) being only $14 \%$. From (5),

$$
R_{0}=\frac{R_{n}}{\beta_{n}}=\frac{1}{\beta_{n}}\left(\frac{I_{0}}{I}\right)_{n}
$$

and, averaging for all spots on the film,

$$
\begin{aligned}
R_{0}= & \frac{\Sigma\left(I_{0} / \beta_{n}\right)}{\Sigma I} \\
& =\frac{\Sigma I_{0}}{\Sigma \beta_{n} I} .
\end{aligned}
$$

$\begin{array}{cccc}\text { as function of } R_{0} \text { and } \zeta & & \\ 2.4 & 2.5 & 2.6 & \text { Average } \beta_{n} \\ 1 & 1 & 1 & 1 \\ 1.004 & 1.005 & 1.005 & 1.00 \\ 1.019 & 1.019 & 1.020 & 1.02 \\ 1.043 & 1.045 & 1.047 & 1.04 \pm 0.01 \\ 1.083 & 1.087 & 1.091 & 1.08 \pm 0.01 \\ 1.145 & 1.153 & 1.160 & 1.14 \pm 0.02 \\ 1.245 & 1.257 & 1.270 & 1.24 \pm 0.04 \\ 1.419 & 1.443 & 1.466 & 1.41 \pm 0.06 \\ 1.793 & 1.843 & 1.891 & 1.77 \pm 0.13\end{array}$

It is preferable to use (8) rather than (9), since (8) gives less weight in averaging to the measurements for which $\beta_{n}$ is high. The high values of $\beta_{n}$ (for high layer lines) are not known so precisely because $(a)$ they depend to a greater degree on the value of $R_{0}$ than do the lower values, and $(b)$ the $\zeta$ values, and consequently the $\beta_{n}$ 's, for high layer lines are known less accurately than for lower layers.

The procedure for finding $R_{0}$ by this method is:

(i) $I_{0}$ and $I$ are measured for each reflexion, within the limits previously defined;

(ii) for each layer in turn, the $I_{0}$ values are totalled and the sum is divided by the 'average $\beta_{n}$ ' appropriate to that $\zeta$ value, giving $\left(\Sigma I_{0}\right)_{n} / \beta_{n}$ for each layer;

(iii) the $\left(\Sigma I_{0}\right)_{n} / \beta_{n}$ values for all layers are totalled, giving $\Sigma\left(I_{0} / \beta_{n}\right)$;

(iv) all the $I$ values are totalled, giving $\Sigma I$;

(v) and, finally, $R_{0}$ is calculated using (8).

When $R_{0}$ has been found, $R_{1}, R_{2}, R_{3}, \ldots$ can be calculated using (5) with the value of $\beta_{n}$ appropriate to $n$ (or $\zeta$ ) and to this $R_{0}$.

The effect discussed above also operates when films are interleaved with metal foils for use with Mo $K$ radiation and the methods given are still applicable. When the equi-inclination Weissenberg method (or any other method in which the layers are recorded separately) is used, the correlating factor will be constant over any one pair of films (for one layer) but will vary from layer to layer. Consequently one cannot assume one average value of the factor for all layers.

When other ways of supporting the photographic films are used, the correlating factor will vary with $\xi$ as well as with $\zeta$ and the treatment will be more complex.

The author wishes to thank Prof. Kathleen Lonsdale for encouragement and advice, and he wishes to acknowledge the award, by the University of London, of a Postgraduate Studentship.

\section{References}

Cox, E. G. \& Shaw, W. F. B. (1930). Proc. Roy. Soc. A, 127, 71 .

QURASHI, M. M. (1953). Acta cryst. 6, 668.

Robertson, J. M. (1943). J. Sci. Instrum. 20, 175.

Whittaker, E. J. W. (1953). Acta Cryst. 6, 218. 